\title{
A New Approach to the Complicated Liver Hydatid Cyst - Laparoscopic Roux-en-Y Cystojejunostomy
}

\section{Huseyin Yilmaz, Mustafa Sahin, Ilhan Ece, Serdar Yormaz, Husnu Alptekin} Department of Surgery, Faculty of Medicine, Selcuk University, Konya, Turkey

Received May 25, 2015; Accepted September 15, 2015.

Key words: Laparoscopy - Hepatic hydatid disease - Cystojejunostomy

\begin{abstract}
Surgery is still the gold standard in the treatment of hepatic hydatid disease. One of the major problems associated with hydatid cyst surgery is biliary fistula. In this report, we aimed to describe a laparoscopic internal drainage method for the treatment of complicated hepatic hydatid cyst in order to prevent long-term biliary fistulas. A 44-year-old female was referred to our clinic with the complaints of flank pain. On computerized tomography of the abdomen, an 8-cm hydatid cyst was detected on the right lob of the liver. Laparoscopic exploration demonstrated a cysto-biliary communication, and laparoscopic cystojejunostomy was performed to prevent long-term bile leakage. The patient was discharged on postoperative day 5 without any problems. In $45^{\text {th }}$ days, nearly complete obliteration of the cavity was observed. Laparoscopic cystojejunostomy can be an effective and safe surgical approach for the treatment of complicated hepatic hydatid cysts.
\end{abstract}

Mailing Address: Ilhan Ece, MD., Department of Surgery, Faculty of Medicine, Selcuk University, Selcuk Universitesi Tıp Fakultesi Alaeddin Keykubat kampusu 42075, Selcuklu, Konya, Turkey; Phone: +90 53255028 62; e-mail: ilhanece@yahoo.com 


\section{Introduction}

Hydatid disease is a chronic parasitic infection caused by the larval stage of Echinococcus granulosus and is endemic in many parts of the world including North Africa, Eastern Europe, Mediterranean countries, Central Asia, and China (Craig et al., 2007). In most of the cases, the disease involves the liver (Pedrosa et al., 2000). A mature cyst consists of a layer of living tissue, which includes the germinal layer that surrounds the fluid-filled central hydatid cavity and the laminated membrane together forming endocyst.

The treatment option for hydatid disease is surgery, drug therapy and percutaneous drainage (Sayek et al., 1980). Over the years, conventional and laparoscopic surgical treatments for hydatid disease have been widely accepted by the experienced surgeons all around the world (Koea, 2012). Recently, a broad spectrum of surgery ranging from simple drainage to radical liver resection by the laparoscopic technique in patients with liver hydatid disease have been reported. The appropriateness of choosing a particular surgical treatment of hepatic hydatidosis depends on relation between the cyst and the bile ducts. In cysts with bile duct communication, the proposed technique of cavity management and biliary

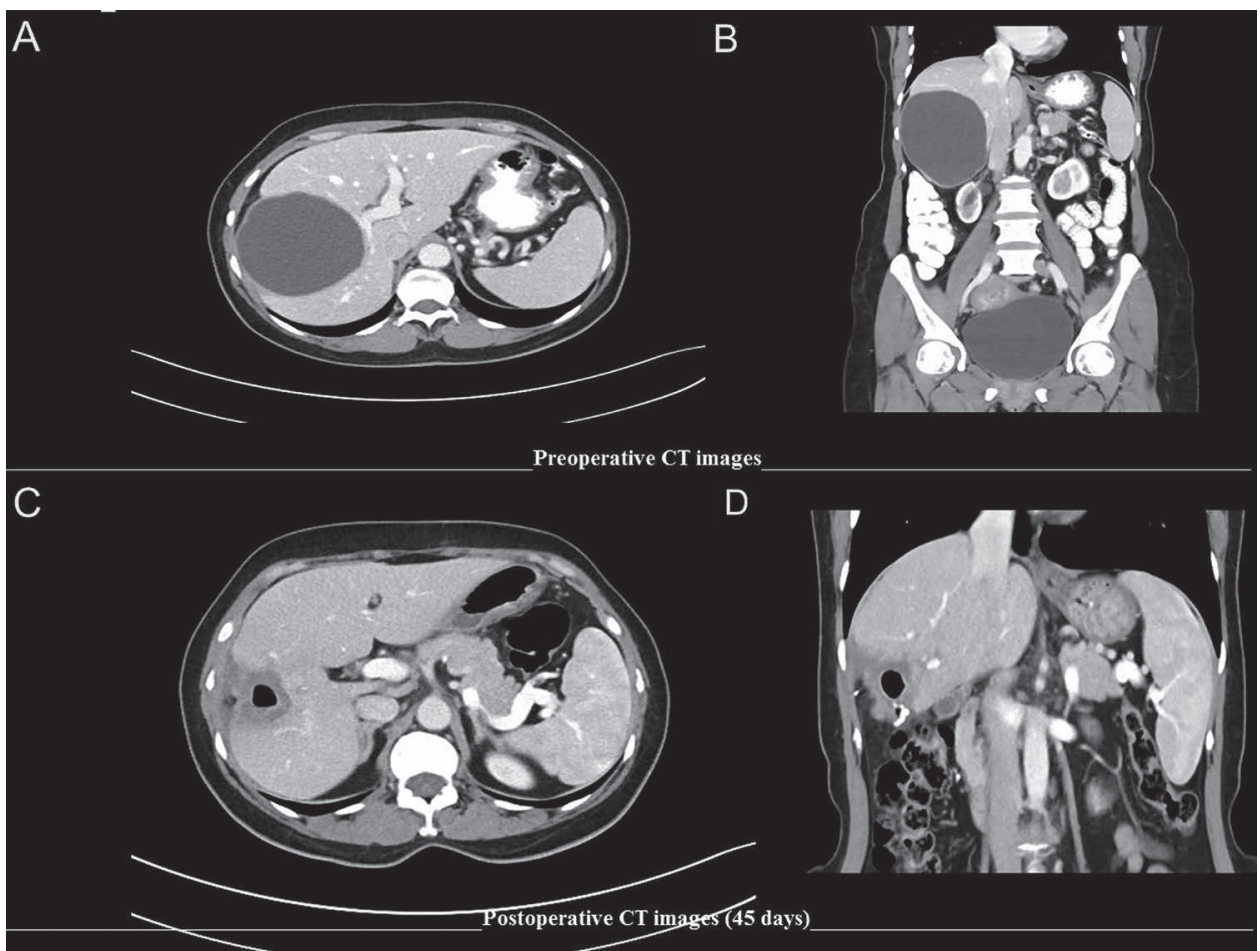

Figure 1 - Preoperative axial (A) and coronal (B) CT images compared with postoperative axial (C) and coronal (D) images. 
drainage procedures vary greatly while the ideal operation is still controversial (Bedirli et al., 2002).

Herein we aimed to report laparoscopic cystotomy and cystojejunostomy performed in a case with hepatic hydatid disease.

\section{Case report}

A 44-year-old woman was admitted to our clinic because of a flank pain. The physical examination was normal. Complete blood count, serum biochemistry and urinary parameters were normal. There was no history of fever, jaundice, or cholangitis, and radiologic evidence of bile duct dilatation as an indicator of cystobiliary communication. Abdominal computed tomography showed an 8 centimeters diameter hydatid cyst located in the segment 7 (Figure 1A and B). Patient received oral albendazole $(10 \mathrm{mg} / \mathrm{kg})$ for 2 weeks before surgery.

\section{Surgical procedure}

Patient received endotracheal general anesthesia and was placed in a 30 degree reverse Trendelberg position with the surgeon standing in between the legs.
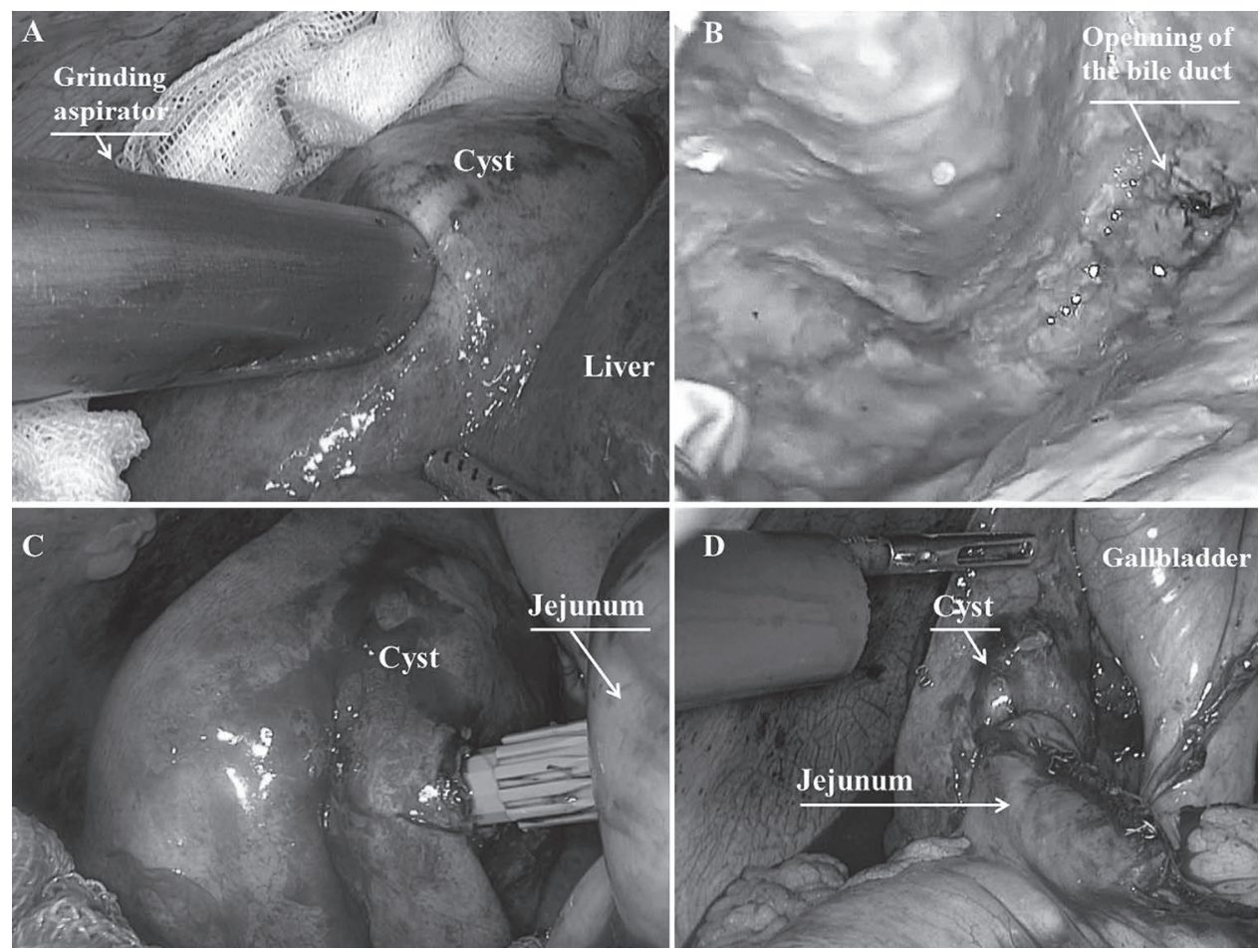

Figure 2 - A grinding aspirator inserted to the cyst (A) and large opening of the bile duct is seen in the cavity (B). An anastomosis performed to the bottom of cyst (C) and distal part of the jejenum closed by a linear cutter (D). 
The first $10-\mathrm{mm}$ trocar was inserted supraumbilically and carbon dioxide pneumoperitoneum was established and intra-abdominal pressure was adjusted at $12 \mathrm{~mm} \mathrm{Hg}$. Thereafter, a 30-degree laparoscope was used to explore the abdominal cavity. After routine exploration, two $10-\mathrm{mm}$ trocars were inserted from right and left midclavicular lines, and 4 pieces of gauze soaked with 20\% sodium chloride were introduced in the abdominal cavity and placed around the cyst and liver to prevent the leakage of the cystic contents. Half of the cyst fluid was aspirated with a Veress needle, scolisidal agent ( $20 \%$ sodium chloride), in the amount of $1 / 2$ volume of aspirated cyst fluid, was injected into the cyst cavity, after waiting 5 minutes, all of cyst fluid was re-aspirated. After inactivation and preventing procedures, a grinding aspiratory was inserted into the cyst cavity from the top of the cyst wall. Cyst contents were ground and aspirated. Cystotomy was performed on the top of the cyst, the cavity cleaned and washed with hypertonic saline for residual daughter vesicles, by brushing with sponges. Then the cavity was inspected carefully for bile duct communication. As a result, large relation with the biliary tract were seen (Figure 2B). Based on the latter findings it was decided to perform the Roux-en-Y cystojejunostomy. An anvil of 28 milimeters circular stapler was inserted in the cyst cavity. Forty centimetres away from the Treitz ligament, an efferent loop was prepared. The stapler itself was placed into the loop. Cystojejunostomy was performed from the bottom of the cyst beside the right side of the gallbladder (Figure 2C and D). Distal end of the jejenum was closed with an endoscopic stapler (Figure 2D). Forty centimetres away from the cystojejunostomy, jejunojejunostomy was performed via endoscopic stapler. The opened part of the cyst was closed with prolen sutures continuously. The patient was discharged on the $5^{\text {th }}$ day without any problem. Forty-five days after the operation, computed tomography showed a cystic mass of about 3 centimeters diameter (Figure 1C and D). The patient was discharged from the hospital with albendazole $400 \mathrm{mg}$ twice a day for six weeks. Six-month follow-up period was uneventful.

\section{Discussion}

The hydatid disease primarily affects the liver. All patients with hydatidosis symptoms should be treated surgically and also asymptomatic patients with cysts greater than $5 \mathrm{~cm}$ in diameter should be considered as surgical conditions (Pedrosa et al., 2000). The first successful laparoscopic resection of liver hydatid disease was performed in 1992 (Katkhouda et al., 1992). An increasing number of laparoscopic treatments of liver hydatid disease have been reported to the present. The reported advantages are minimal invasiveness, more detailed cyst inspection, reduced wound complication, reduced hospital stay, and cost effectiveness (Avgerinos et al., 2006). Different therapeutic modalities including simple drainage, cystectomy, pericystectomy, and radical liver resection have been successfully performed by the laparoscopic technique. Laparoscopy began to be 
widely accepted as the first choice of treatment in selected liver hydatid patients (Palanivelu et al., 2006).

Radical methods have several advantages such as reduced incidence of local recurrence, avoiding usage of drug therapy, better management of cysto-biliary communications, reducing rate of biliary fistula, and cavity-related complications and recurrence. Cavity-related complications including the biliary leakage, biliary fistula and intraabdominal abscess are the main problems in the conservative surgical methods of hydatid disease. One of the operative procedures for obliteration of the residual cavity can be selected according to the thickness of the pericyst wall, size of cavity, severity of infection, and degree of bile leakage (Xu, 1992). Although the partial cystectomy and unroofing are the most widely used techniques, many complications can be faced especially in cases with biliary tract communications. Roux-en-Y cystojejunostomy with open procedure has been used for complicated hydatid cysts for a long time (Acar et al., 2014). However the laparoscopic Roux-en-Y cystojejunostomy is not included in the current literature.

Hydatid cysts may be located in the central and peripheral parts of the liver, and treatment varies depending on the location of the cyst. The fistulous openings detected in peripherally located cysts should be sutured to prevent complications during cystotomy or partial cystectomy, however small biliary fistulas may be left alone. Centrally located cysts with bile duct communication can be more difficult to treat. Cyst may erode the lateral side of the bile duct wall. In this instance, proximal and distal end of bile duct can be seen in the cystic cavity. Proximal opening can be sutured but suturing the distal opening disrupts the drainage of the remaining liver segments. Therefore the treatment of centrally located cysts is the most unique indication of this technique. The majority of biliary leakages can close spontaneously within several days (Yuksel et al., 2008; Symeonidis et al., 2013). Nevertheless, the major problem is reported to be the long-term biliary fistula. In this case, eroded biliary ducts with largely open draining to the cyst cavity were visible. In order to minimize the risk of probable biliary fistula, an internal drainage technique was performed laparoscopically. We performed a Roux-en-Y cystojejunostomy at the right side of the gallbladder for a cyst settled in the segment 7 of the liver. The anastomosis was performed to the bottom of the cyst in order to ensure full drainage. In this way, the higher reduction of the cyst cavity is possible while the loss of bile and electrolytes through intestinal drainage is avoided. There was no electrolyte imbalance during six-month follow-up time.

The presence of a history of biliary colic, preoperative jaundice or obstructive liver function tests, radiological evidence of biliary dilatation or pneumobilia are all highly suggestive of a biliary fistula. It is recommended that these cases be underwent pre-intraoperative cholangiography and more conventional open surgery. In our clinical practice; symptomatic patients with cholestasis were evaluated with magnetic resonance cholangiopancreatography preoperatively. In 
this case, there is no evidence of intrahepatic cholestasis to perform the pre-and intraoperative imaging.

As a result, laparoscopic Roux-en-Y cystojejunostomy is an effective and safe surgical approach for the treatment of centrally located hepatic hydatid cysts, with a lower rate of morbidity, and thus may become the surgical treatment of choice for hepatic hydatid cysts with bile duct communication.

\section{References}

Acar, F., Sahin, M., Alptekin, H., Yılmaz, H., Ertuğrul Kafalı, M. (2014) Surgical treatment of giant liver hydatid cysts: Comparison of cystojejunostomy and partial cystectomy. Surg. Today 44(11), 2065-2071.

Avgerinos, E. D., Pavlakis, E., Stathoulopoulos, A. (2006) Clinical presentations and surgical management of liver hydatidosis: our 20-year experience. HPB (Oxford) 8, 189-193.

Bedirli, A., Sakrak, O., Sozuer, E. M., Kerek, M., Ince, O. (2002) Surgical management of spontaneous intrabiliary rupture of hydatid liver cysts. Surg. Today 32(7), 594-597.

Craig, P. S., McManus, D. P., Lightowlers, M.W., Chabalgoity, J. A., Garcia, H. H., Gavidia, C. M., Gilman, R. H., Gonzalez, A. E., Lorca, M., Naquira, C., Nieto, A., Schantz, P. M. (2007) Prevention and control of cystic echinococcosis. Lancet Infect. Dis. 7, 385-394.

Katkhouda, N., Fabiani, P., Benizri, E., Mouiel, J. (1992) Laser resection of a liver hydatid cyst under videolaparoscopy. Br. J. Surg. 79, 560-561.

Koea, J. B. (2012) Laparoscopic treatment of hepatic hydatid disease. ANZ J. Surg. 82(7-8), 499-504.

Palanivelu, C., Jani, K., Malladi, V., Senthilkumar, R., Rajan, P. S., Sendhilkumar, K., Parthasarthi, R., Kavalakat, A. (2006) Laparoscopic management of hepatic hydatid disease. JSLS 10, 56-62.

Pedrosa, I., Saiz, A., Arrazola, J., Ferreiros, J., Pedrosa, C. S. (2000) Hydatid disease: Radiologic and pathologic features and complications. Radiographics 20(3), 795-817.

Sayek, I., Yalin, R., Sanac, Y. (1980) Surgical treatment of hydatid disease of the liver. Arch. Surg. 115, 847-850. Symeonidis, N., Pavlidis, T., Baltatzis, M., Ballas, K., Psarras, K., Marakis, G., Sakantamis, A. (2013) Complicated liver echinococcosis: 30 years of experience from an endemic area. Scand. J. Surg. 102(3), 171-177.

Xu, M. Q. (1992) Diagnosis and management of hepatic hydatidosis complicated with biliary fistula. Chin. Med. J. 105(1), 69-72.

Yuksel, O., Akyürek, N., Sahin, T., Salman, B., Azili, C., Bostanci, H. (2008) Efficacy of radical surgery in preventing early local recurrence and cavity-related complications in hydatic liver disease. J. Gastrointest. Surg. 12(3), 483-489. 